\title{
Quantitative measurement of cast metal relics by pulsed
}

\section{thermal imaging}

\author{
Ning Tao, Jiangang Sun, Congsi Wang, Cunlin Zhang \\ Beijing Key Lab for Terahertz Spectroscopy and Imaging, Key Lab of Terahertz Optoelectronics, \\ Ministry of Education, and Beijing Advanced Innovation Center for Imaging Theory and \\ Technology, Department of Physics, Capital Normal University, Beijing 100048, China
}

\begin{abstract}
Corrosion monitoring and detection of cast metal relics has always been a concern of ancient culture relics conservation and restoration researchers. In recent years, there is increased interest in the determination of the corrosion thickness and matrix thickness of bronze and iron cultural relics. Due to the interaction of metal materials and atmospheric, water, soil or pollutant deposition, or the tremendous changes in the surrounding environment before and after the excavation of cultural relics, most of the metal cultural relics will be corroded to varying degrees, and even worsened. Therefore, it is necessary to measure the loss of surface materials or corrosion thickness of artworks in the process of corrosion. On the one hand, the detection of corrosion state or corrosion thickness can help to predict the long-term degradation trend of metal artworks as well as to assess the deterioration process. On the other hand, it is more meaningful to formulate plans for the protection and restoration of cultural relics. Traditional non-destructive detection techniques, such as ultrasound or X-ray, may be time-consuming or inconvenient. A safe, fast, non-contact and good sensitive detection method would be a better choice. In this paper, an investigation on detection and measurement of cast metal relics corrosion by pulsed thermal imaging is carried out. Not only the information of corrosion thickness but also the substrate thickness is of great significance to the monitoring of corrosion status, rust cleaning, restoration and conservation of such cultural relics. Current thickness measurement methods of pulsed thermal imaging are mostly aimed at a single layer material such as flat-bottom holes material or a semi-infinite two-layer material, without considering the effect of the finite substrate thickness, which could affect to the accuracy of the predicted first layer thickness. Nor is it possible to measure the thickness of the substrate metal material, the second layer. This paper presents a new method for measurement of the corrosion thickness and matrix thickness of a replica of cast iron Buddha head which based on the pulsed thermal imaging - multilayer analysis (PTI-MLA) method. This method has been commonly used for measuring of coating material thermal properties and for the first time it has been used in quantitative measurement in culture relics. PTI employs a simple
\end{abstract}


one-sided test system, consists of an commercial FLIR-A6751 infrared (IR) camera, two flash lamp with totally 4800J power, an IR filter, a modern imitation cast iron Buddha head sample, and a data acquisition and processing computer. MLA is an automated data processing program to determine thermal properties or thickness of multilayer materials based on a regression algorithm. Firstly, a one-sided pulsed thermal imaging experiment was set up and applied to obtain the surface temperature of cast iron Buddha head from four angles through 90 degree rotation separately. In this study, the thermal diffusivity of cast iron is used as a known parameter to measure the thickness of substrate, so we first measured the overall thickness of the measurable area of Buddha head and calculated the thermal diffusivity of cast iron by the well-established Flash method using the same experimental instruments. After that the corrosion thickness and substrate thickness of cast iron Buddha head were measured by non-linear fitting of theoretical solution and experimental value. After all the pixels are operated on the same way, the images of corrosion thickness and substrate thickness are obtained. Then the different finite substrate thickness effect on the corrosion thickness measurement is analyzed by theoretical simulation. We also calculated the overall thickness distribution of the Buddha head with the measured thermal diffusivity of cast iron by using the flash method. Finally the experimental results were discussed and reasonable. The calculated thickness of cast iron Buddha head by flash method and PTI-MLA method are basically consistent. The measurement results show that this new method has great potential and capability in quantitative measurement of ancient bronze and iron cultural relics. 